\title{
The Comparative Study of the Temperature Distribution of Fiber Laser with Different Pump Schemes
}

\author{
Mostafa Abouricha ${ }^{1 *}$, Abdelkader Boulezhar ${ }^{1}$, Nabil Habiballah ${ }^{2 *}$ \\ ${ }^{1}$ Hassan II Casablanca University, Faculty of Sciences-Ain Chok, \\ Laboratory of Theoretical and Applied Physics, Casablanca, Morocco \\ ${ }^{2}$ Chouaib Doukkali University, Faculty of Science, Laboratory of Condensed Matter of Physics, El Jadida, Morocco \\ Email: ${ }^{*}$ mmabouricha@yahoo.fr, ${ }^{*}$ n.habib1980@gmail.com
}

Received August 6, 2013; revised September 6, 2013; accepted October 13, 2013

Copyright (C) 2013 Mostafa Abouricha et al. This is an open access article distributed under the Creative Commons Attribution License, which permits unrestricted use, distribution, and reproduction in any medium, provided the original work is properly cited.

\begin{abstract}
Based on the structure of the long fiber laser (YDCFLs) with different pump schemes using high pump power, the nonlinear coupled and heat dissipation equations are solved numerically. Using the finite-difference method, we have determined the temperature distribution along the radial and axial directions of the fiber laser (YDCFLs) for the forward pump schemes of $200 \mathrm{~W}$ with reflection $R_{p 2}$, backward pump schemes of $200 \mathrm{~W}$ with reflection $R_{p 1}$ and for bidirectional pump scheme of $100 \mathrm{~W}$ each side. The results are: the temperature distribution for bidirectional pump mode is more even than that for forward pump with reflection $R_{p 2}$ and than that for backward pump with reflection $R_{p 1}$. The results show that the maximum temperature difference between different schemes is $57.51^{\circ} \mathrm{C}$, and when the air-clad width decreases, the temperature in the core regions also decreases and does not affect to the cladding radius regions. We summarize that the temperature in the core and in cladding radius regions decreases when the outer radius cladding increases.
\end{abstract}

Keywords: Pump Scheme; High Power Fiber Laser; Temperature Distribution

\section{Introduction}

The double-clad fiber laser (DCFLs) have several applications in modern telecommunication, medical instruments, military, defense and material surface processing, because of some unique advantages including high conversion efficiency, excellent beam quality, less thermal effect [1-3]. The continuous wave emission up to kWlevel has been reported $[4,5]$. A new type of photonic crystal fibers (PCFs) has opened numerous axes in research; this is due to its unique properties such as endlessness.

Thermal effect in Single mode guiding in high-power fiber laser has attracted much attention in recent days [6, 7]. Also, the developments of cladding-pumped fiber technology and high brightness semiconductor diode pump lasers enabled have been used to save energy [8]. The evolution of the temperature and the influence of the core, inner-clad, air-clad and outer clad, on the shape of optical fiber have been studied [9].

Numerically, Shang [8] has investigated the output power characteristics of YDCFLS with different pump

${ }^{*}$ Corresponding authors. schemes, finding the bidirectional pump scheme is compatible with the backward with $R_{p 1}=0$ and forward pump scheme with $R_{p 2}=0$. However, the backward pumped YDCFL with $R_{p 1}=0.98$ has the highest conversion efficiency; while the forward pumped YDCFL with $R_{p 2}=0.98$ has maximum output power per meter.

In this paper, we have investigated numerically the theoretical and numerical analysis of 2D Temperature field by solving the transient heat conduction equations comparing in detail the temperature distributions in the backward pump schemes with $R_{p 1}$, forward pump schemes with $R_{p 2}$ and bidirectional pump scheme. The results show that the maximum temperature in the two-end pump is $96.89^{\circ} \mathrm{C}$, in the forward pump is $148.4^{\circ} \mathrm{C}$ and in the backward pump is $154.4^{\circ} \mathrm{C}$. Thus, the temperature distributions for two-end pump mode is more even than that for forward pump with reflection $R_{p 2}=0.98$ and backward pump with reflection $R_{p 1}=0.98$ mode.

\section{Theoretical Analysis}

Figure 1 demonstrates the schematic of YDCFL. For the convenience of analysis, the pump light and output laser 


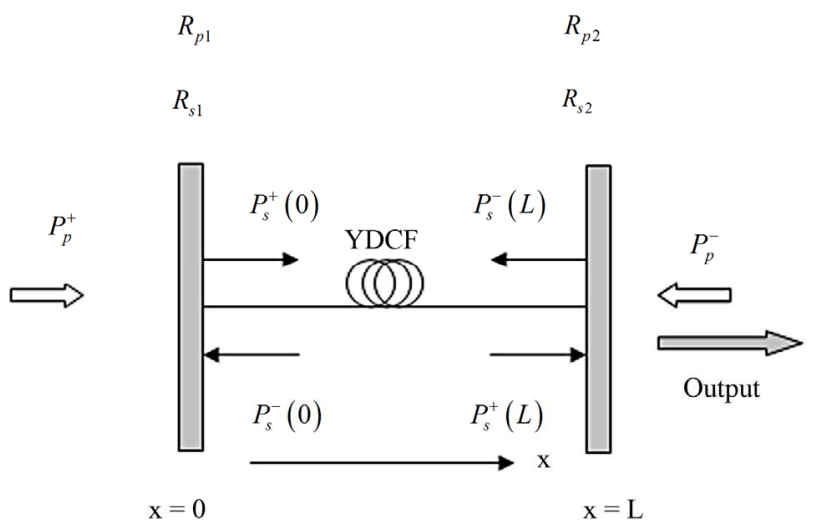

Figure 1. Schematic demonstration of ytterbium-doped double-clad fiber laser.

are assumed to be narrow spectra and regarded as single wavelength, ignoring the non linear effects and influences of amplified spontaneous emission on the inverse population. Using the rate-equation theory under the steady state conditions, we can be governed the propagations of the light wave power in fiber cavity as follows [7]:

$$
\begin{aligned}
& \pm \frac{P_{p}^{ \pm}(x, t)}{\partial x}=\Gamma_{p}\left[\left(\sigma_{a p}+\sigma_{e p}\right) N_{b}(x, t)-\sigma_{a p} N_{y t}\right] \\
& \times P_{p}^{ \pm}(x, t)-\alpha_{p} P_{p}^{ \pm}(x, t) \\
& \pm \frac{P_{s}^{ \pm}(x, t)}{\partial x}=-\Gamma_{s}\left[\left(\sigma_{a s}+\sigma_{e s}\right) N_{b}(x, t)-\sigma_{a s} N_{y t}\right] \\
& \times P_{s}^{ \pm}(x, t)-\alpha_{s} P_{s}^{ \pm}(x, t) \\
& \frac{N_{b}(x, t)}{\partial t} \\
& =\left(\frac{\lambda_{p} \Gamma_{p} \sigma_{a p}}{h c A_{c o}}\right)\left[N_{y t}-N_{b}(x, t)\right]\left[P_{p}^{+}(x, t)+P_{p}^{-}(, t)\right] \\
& -\left(\frac{\lambda_{p} \Gamma_{p} \sigma_{a p}}{h c A_{c o}}\right)\left[N_{y t}-N_{b}(x, t)\right]-\left(\frac{\lambda_{p} \Gamma_{p} \sigma_{a p}}{h c A_{c o}}\right) \\
& \cdot\left[N_{y t}-N_{b}(x, t)\right]\left[P_{p}^{+}(x, t)+P_{p}^{-}(, t)\right] \\
& -\left(\frac{\lambda_{p} \Gamma_{p} \sigma_{a p}}{h c A_{c o}}\right)\left[N_{y t}-N_{b}(x, t)\right]\left[P_{p}^{+}(x, t)+P_{p}^{-}(, t)\right] \\
& -\frac{N_{b}(x, t)}{\tau}
\end{aligned}
$$

Equations (1)-(3), $N_{y t}$ is the concentration of $\mathrm{Yb}^{3+}$, $N_{b}(x, t)$ is the upper laser level population density, $P_{p}^{+}(x, t)$ and $P_{p}^{-}(x, t)$ represent the power of forward and backward propagation pump light, respectively. $P_{s}^{+}(x, t)$ and $P_{s}^{-}(x, t)$ represent the power of forward and backward propagation laser power, respectively. $\Gamma_{p}$ and $\Gamma_{s}$ are power filling factors. $\sigma_{a s}$ and $\sigma_{e s}$ are the laser absorption and emission cross section, $\sigma_{a p}$ and $\sigma_{e p}$ are the pump light absorption and emission cross-section respec- tively. $\tau$ is the lifetime of $Y b^{3+}$ in upper level, $\alpha_{p}$ and $\alpha_{s}$ represent scattering loss coefficients of laser light and pump light respectively. The above Equations (1)-(3) at fiber front $(x=0)$ and fiber rear end $(x=L)$ satisfy the boundary conditions for the laser as follows:

$$
P_{s}^{+}(0)=R_{s 1} P_{s}^{-}(0), \quad P_{s}^{-}(L)=R_{s 2} P_{s}^{+}(L)
$$

Where $R_{s 1}=0.98$ and $R_{s 2}=0.04$ the power laser reflectively of the reflector at $x=0$ and $x=L$, respectively. And the boundary conditions for the pump propagation equation can be expressed as follows [8]:

$$
\begin{gathered}
P_{p}^{+}(0)=P_{p 01}, \quad P_{p}^{-}(L)=R_{p 2} P_{p}^{-}(L) \\
P_{p}^{-}(L)=P_{p 02}, \quad P_{p}^{+}(0)=R_{p 1} P_{p}^{-}(0) \\
P_{p}^{+}(0)=P_{p 01}, \quad P_{p}^{-}(L)=P_{p 02},
\end{gathered}
$$

Where $P_{p 01}$ the launched pump power into the front end $(x=0)$ and $P_{p 02}$ the launched pump power into the back end $(x=L) . R_{p 2}$ and $R_{p 1}$ represent the reflectivity at pump wave length in forward and backward pumped fiber laser, respectively.

In the YDCF, heat is generated in the fiber core, and this heat is transferred from the fiber core to the inner clad by thermal conduction, and by thermal conduction and radiation to air-clad, in air clad no convection because the width air-clad is very small, which justified by Grashof number $G_{r \delta}$ in air clad given by [10] and by thermal conduction and radiation to other clad, and dissipated from the fiber surface to ambient air by convective and radiative heat transfer. Therefore the transverse temperature distributions in the YDCF at room temperature are governed by following thermal conductive equations in symmetric cylindrical coordinates [11].

$$
\nabla(-k \nabla T)=Q(x)
$$

Then we find:

$$
\begin{gathered}
\frac{1}{r} \frac{\partial}{\partial r}\left(r \frac{\partial T_{1}(r)}{\partial r}\right)=-\frac{Q(x)}{k_{1}},(0 \leq r \leq a) \\
\frac{1}{r} \frac{\partial}{\partial r}\left(r \frac{\partial T_{2}(r)}{\partial r}\right)=0,\left(a \leq r \leq b_{1}\right) \\
\frac{1}{r} \frac{\partial}{\partial r}\left(r \frac{\partial T_{3}(r)}{\partial r}\right)=0, \quad\left(b_{1} \leq r \leq b_{2}\right) \\
\frac{1}{r} \frac{\partial}{\partial r}\left(r \frac{\partial T_{4}(r)}{\partial r}\right)=0,\left(b_{2} \leq r \leq b\right)
\end{gathered}
$$

Where $a, b_{1}, b_{2}$ and $b$ represent the radii of the core, inner clad, air-clad and outer clad respectively. $T_{1}(r)$, $T_{2}(r), T_{3}(r)$ and $T_{4}(r)$ represent the temperature in fiber core, inner clad, air-clad and outer clad, respectively. $Q(x)$ represents the heat power density along the axial direc- 
tion of the fiber core. Which mainly depends on the scattering and loss of pump light and given by:

$$
Q(x)=\frac{\alpha(x)\left(P_{p}^{+}(x, t)+P_{p}^{-}(x, t)\right)}{\pi a^{2}}(1-S)
$$

Where $\alpha(x)=\alpha_{\alpha}(x)+\alpha_{p}, \alpha_{\alpha}(x)$ is absorption coefficient, and $S$ is the quantum efficiency whose theoretical value is $\lambda_{p} / \lambda_{s}$. However, it cannot reach the theoretical value in practical applications. In Other regions of the fiber, the value of $Q(x)$ is zero. Supposing perfect thermal connection among the cladding and inner core, the temperature and their derivatives are continuous at the boundary $(r=a)$, therefore, the boundary conditions can be expressed as follows [12]:

For $r=0$ we have:

$$
\frac{\partial T_{1}(r)}{\partial r}=0
$$

For $r=\alpha$ we have:

$$
T_{1}(r)=T_{2}(r), \quad \frac{\partial T_{1}(r)}{\partial r}=\frac{\partial T_{2}(r)}{\partial r},
$$

For $r=b_{1}$ we have:

$$
\begin{aligned}
& -k_{1} \frac{\partial T_{2}(r)}{\partial r}=-k_{2} \frac{\partial T_{3}(r)}{r \partial}+\varepsilon \sigma\left(T_{c}^{4}-T_{3}(r)^{4}\right), \\
& T_{2}\left(b_{1}\right)=T_{3}\left(b_{1}\right)
\end{aligned}
$$

For $r=b_{2}$ we have:

$$
\begin{aligned}
& -k_{2} \frac{\partial T_{3}(r)}{\partial r}+\varepsilon \sigma\left(T_{c}^{4}-T_{3}(r)^{4}\right)=-k_{1} \frac{\partial T_{4}(r)}{\partial r}, \\
& T_{3}\left(b_{2}\right)=T_{4}\left(b_{2}\right)
\end{aligned}
$$

In Figure 2, we have demonstrates the temperature difference between the ambient air and the fiber surface is determined by the convective and radiation heat flow, and thus the boundary condition cross the fiber other cladding and the ambient air is given by the Newton's law and the Stefan-Boltzmann law [13].

$$
\frac{\partial T_{4}(r)}{\partial r} \|_{r=b}=\frac{h_{c}}{k_{2}}\left[T_{c}-T_{4}(b)\right]+\varepsilon \sigma\left(T_{c}^{4}-T_{4}(b)^{4}\right),
$$

Where $k_{2}$ is the thermal conductivity of air, $k_{1}$ is the thermal conductivity of material, $T_{c}$ is the temperature of ambient air, $\varepsilon$ is the emissivity, $\sigma$ is the Stefan-Boltzman constant and $h_{c}$ is the convective coefficient which can be calculated by [14].

$$
\begin{gathered}
G r=\frac{g a b^{3}\left(T_{c}-T\right)}{v^{2}}, a=\left(\frac{T_{c}-T}{2}\right)^{-1}, \quad R_{a}=G r P_{r}, \\
N_{u}=\left\{0.60+\frac{0.387 R_{a}^{1 / 6}}{\left[1+\left(0.556 / P_{r}\right)^{9 / 16}\right]^{8 / 27}}\right\}^{2}
\end{gathered}
$$

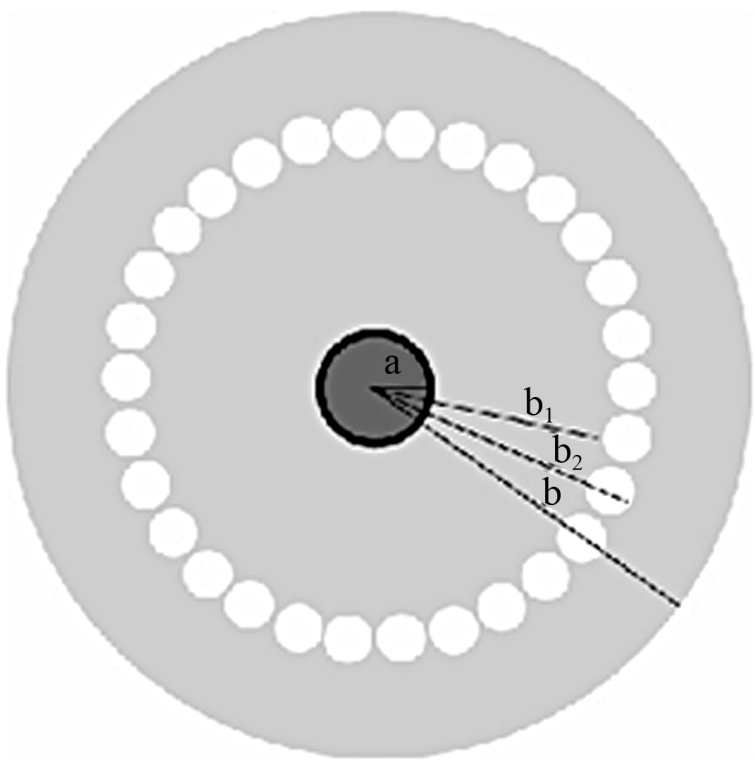

Figure 2. Heat dissipation model of PCF [9].

$$
h=0.5 N_{u} b^{-1},
$$

Where $N_{u}, P_{r}$ and $G r$ are Nusselt, Prandtl numbers and Grashof, respectively. $g$ and $v$ are the acceleration and Kinematic viscosity, the temperature in regions core, inner clad, air-clad and other clad can be derived by using Equations (9)-(12) subject to the boundary conditions (14)-(17) as follows:

$$
\begin{gathered}
T_{1}(r)=T_{0}-\frac{Q(x) \times r^{2}}{4 k_{1}}, \quad \text { if }(0 \leq r \leq a) \quad(22) \\
T_{2}(r)=T_{0}-\frac{Q(x) \times a^{2}}{4 k_{1}}-\frac{Q(x) a^{2} \ln \left(\frac{r}{a}\right)}{2 k_{1}}, \text { if }\left(a \leq r \leq b_{1}\right) \\
T_{3}(r)= \\
T_{2}\left(b_{1}\right)+\left[-\frac{Q(x) a^{2}}{2 k_{2}}+\frac{\varepsilon \sigma b_{1}}{k_{2}}\left(T_{c}^{4}-T_{2}\left(b_{1}\right)^{4}\right)\right] \\
\times \ln \left(\frac{r}{b_{1}}\right), \text { if }\left(b_{1} \leq r \leq b_{2}\right) \\
T_{4}(r)=T_{3}\left(b_{2}\right)+\left[\frac{k_{2}}{k_{1}}\left(-\frac{Q(x) a^{2}}{2 k_{2}}+\frac{\varepsilon \sigma b_{1}}{k_{2}}\left(T_{c}^{4}-T_{2}\left(b_{1}\right)^{4}\right)\right)\right. \\
\left.-\frac{\varepsilon \sigma b_{2}}{k_{1}}\left(T_{c}^{4}-T_{3}\left(b_{2}\right)^{4}\right)\right] \times \ln \left(\frac{r}{b_{2}}\right), \text { if }\left(b_{2} \leq r \leq b\right)
\end{gathered}
$$

Where $T_{0}$ represents the temperature of fiber axis $(r=$ $0)$. Using Equations (22)-(25) subject to boundary condition (18), the relationship between the temperature of fiber surface $T_{4}(b)$ and the ambient air $T_{c}$ is given by Equations (26): 


$$
\begin{aligned}
& {\left[\frac{k_{2}}{k_{1}}\left(-\frac{Q(x) a^{2}}{2 k_{2}}+\frac{\varepsilon \sigma b_{1}}{k_{2}}\left(T_{c}^{4}-T_{2}\left(b_{1}\right)^{4}\right)\right)-\frac{\varepsilon \sigma b_{2}}{k_{1}}\left(T_{c}^{4}-T_{3}\left(b_{2}\right)^{4}\right)\right] } \\
= & \frac{h_{c}}{k_{2}}\left[T_{c}-\left(T_{2}\left(b_{2}\right)+\left[\frac{k_{2}}{k_{1}}\left(-\frac{Q(x) a^{2}}{2 k_{2}}+\frac{\varepsilon \sigma b_{1}}{k_{2}}\left(T_{c}^{4}-T_{2}\left(b_{1}\right)^{4}\right)\right)-\frac{\varepsilon \sigma b_{2}}{k_{1}}\left(T_{c}^{4}-T_{3}\left(b_{2}\right)^{4}\right)\right] \times \ln \left(\frac{b}{b_{2}}\right)\right)\right] \\
& +\varepsilon \sigma\left[T_{c}^{4}-\left(T_{3}\left(b_{2}\right)+\left[\frac{k_{2}}{k_{1}}\left(-\frac{Q(x) a^{2}}{2 k_{2}}+\frac{\varepsilon \sigma b_{1}}{k_{2}}\left(T_{c}^{4}-T_{2}\left(b_{1}\right)^{4}\right)\right)-\frac{\varepsilon \sigma b_{2}}{k_{1}}\left(T_{c}^{4}-T_{3}\left(b_{2}\right)^{4}\right)\right] \ln \left(\frac{b}{b_{2}}\right)\right)^{4}\right]
\end{aligned}
$$

Substituting Equation (26) into Equations (22)-(25), we can obtain the temperature distributions in fiber core and other regions.

\section{Simulation and Discussion}

In our simulation, we have used the Common parameters given in the Table 1. Figure 3 illustrates the output power as a function of fiber length (YDCL) for different pump schemes. It is shown that the increasing output power reaches the maximum value $174.7 \mathrm{~W}$, for length $l$ $=5 \mathrm{~m}$ in the forward pump scheme with reflection $R_{p 2}=$ 0.98 . In the backward pump scheme with reflection $R_{p 1}=$ 0.98 the value reached is $178.7 \mathrm{~W}$ for the length $l=6.4$ $\mathrm{m}$, the maximum value $171.7 \mathrm{~W}$, for length $l=7.4 \mathrm{~m}$ in the forward pump scheme with reflection $R_{p 2}=0$, in the backward pump scheme with reflection $R_{p 1}=0$, the value

Table 1. Common Parameters Used in the Simulation.

\begin{tabular}{cc}
\hline Parameter & value \\
\hline$N_{y t}$ & $1.6 \times 10^{26} \mathrm{~m}^{-3}$ \\
$\lambda_{s}$ & $1080 \mathrm{~nm}$ \\
$\lambda_{p}$ & $975 \mathrm{~nm}$ \\
$A_{e f f}$ & $7.85 \times 10^{-11} \mathrm{~m}^{2}$ \\
$\sigma_{e p}$ & $2.5 \times 10^{-24} \mathrm{~m}^{2}$ \\
$\sigma_{a p}$ & $2.5 \times 10^{-25} \mathrm{~m}^{2}$ \\
$\sigma_{a s}$ & $1.6 \times 10^{-25} \mathrm{~m}^{2}$ \\
$\sigma_{e s}$ & $2.5 \times 10^{-25} \mathrm{~m}^{2}$ \\
$\Gamma_{p}$ & 0.0016 \\
$\Gamma_{s}$ & 0.81 \\
$\tau$ & $1 \mathrm{~ms}$ \\
$\alpha_{s}$ & $5 \times 10^{-3}$ \\
$\alpha_{p}$ & $3 \times 10^{-3}$ \\
$R_{s 1}$ & 0.98 \\
$R_{s 2}$ & 0.04 \\
$\sigma$ & $5.67 \times 10^{-8} \mathrm{w} /\left(\mathrm{m}^{2} \cdot \mathrm{k}^{4}\right)$ \\
$P_{r}$ & 0.701 \\
$g$ & $9.8 \mathrm{~m} / \mathrm{s}^{2}$ \\
$v$ & $1.6 \times 10^{-5} \mathrm{~m}^{2} / \mathrm{s}$ \\
$T_{c}$ & $298 \mathrm{~K}$ \\
$\varepsilon$ & 0.95 \\
\hline & \\
\hline &
\end{tabular}

reached is $177.7 \mathrm{~W}$ for the length $l=10.1 \mathrm{~m}$ and it is reaches the value $174.7 \mathrm{~W}$ for $l=10 \mathrm{~m}$ in the bidirectional pump scheme. Therefore, the forward pump with reflection is in good scheme to minimize the length of YDCF and the backward pump with reflection is the most suitable scheme to minimize the pumping energy.

The heat dissipation along axial, calculated by using Equation (13) and the axial distribution of temperature $(r$ $=0$ ) for different pump schemes have reported, respectively in Figures $\mathbf{4}$ and $\mathbf{5}$.

It is shown that the maximum temperature for the bidirectional pump scheme $T_{\max }=90.89^{\circ} \mathrm{C}$ is lower than that for the forward pump with (reflection $R_{p 2}=0.98$ and $\left.R_{p 2}=0\right)$ as $T_{\max }=148.4^{\circ} \mathrm{C}$ and than that the backward with (reflection $R_{p 1}=0.98$ and $R_{p 1}=0$ ) as $T_{\max }=154.4^{\circ} \mathrm{C}$. The maximum difference is $57.51^{\circ} \mathrm{C}$.

The difference between the maximum and the minimum is: $40.14^{\circ} \mathrm{C}$ in the case of bi-directional pumping, $109.19^{\circ} \mathrm{C}$ in the case of pumping forward with $R_{p 2}=0.98$, $115.38^{\circ} \mathrm{C}$ in the case forward with $R_{p 2}=0,115.19^{\circ} \mathrm{C}$ in the case backward with $R_{p 1}=0.98$ and $121.12^{\circ} \mathrm{C}$ in the case back with $R_{p 1}=0$. Therefore, the reflectivity of the pump has a role crucial is to minimize the temperature difference.

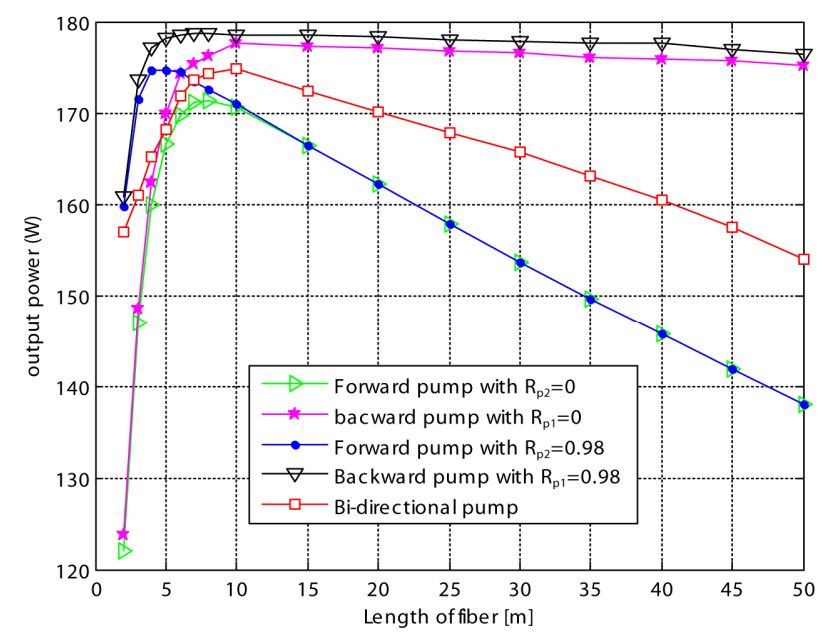

Figure 3. Evolution of output powers a function the YDCF length in different pump schemes for the launched pump power of $200 \mathrm{~W}$. 


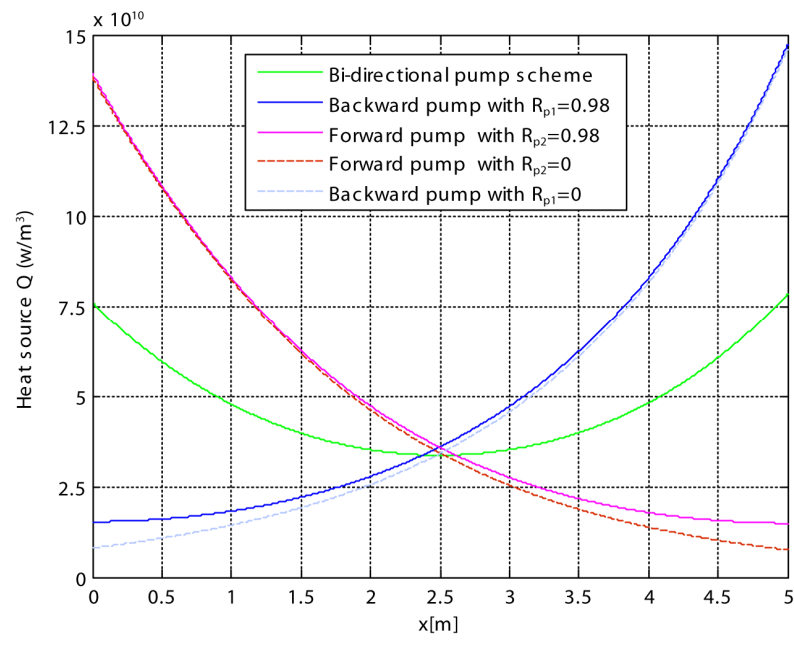

Figure 4. Evolution of heat power density along the fiber in forward pump with $R_{p 2}=0.98$, backward pump with $R_{p 1}=$ 0.98 , forward pump with $R_{p 2}=0$, backward pump with $R_{p 1}$ $=0$ and bi-directional pump mode.

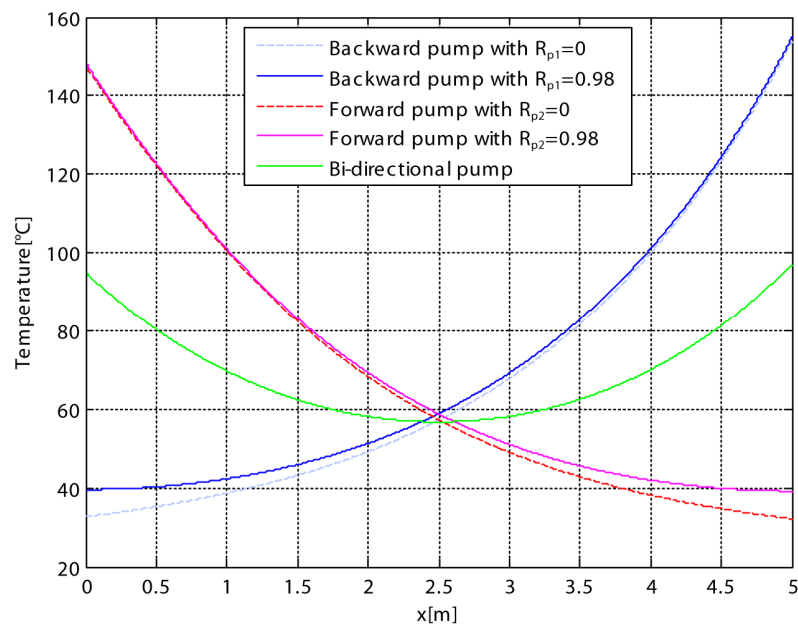

Figure 5. Temperature distribution along the fiber in forward pump with $R_{p 2}=0.98$, forward pump with $R_{p 2}=0$, backward pump with $R_{p 1}=0.98$, backward pump with $R_{p 1}=$ 0 and bi-directional pump mode.

The radial distribution of the temperature is reported in Figures 6-10 as function of core radius for different pump schemes. Consequently, the effect of the radius core is negligible and the maximum temperature difference between $a=5$ and $a=10 \mu \mathrm{m}$ does not exceed $1^{\circ} \mathrm{C}$ in $(r=0)$ for different pump schemes Figures (a). But in Figures (b), we have shown that the temperature distributions at the fiber in $(r=0)$ increases when the air-clad increases for different pump schemes. In Figures (c), we can see another scenario as the temperature of the fiber increases when the outer cladding radius increases.

\section{Conclusion}

In this paper, we have compared in detail the heat dissi-

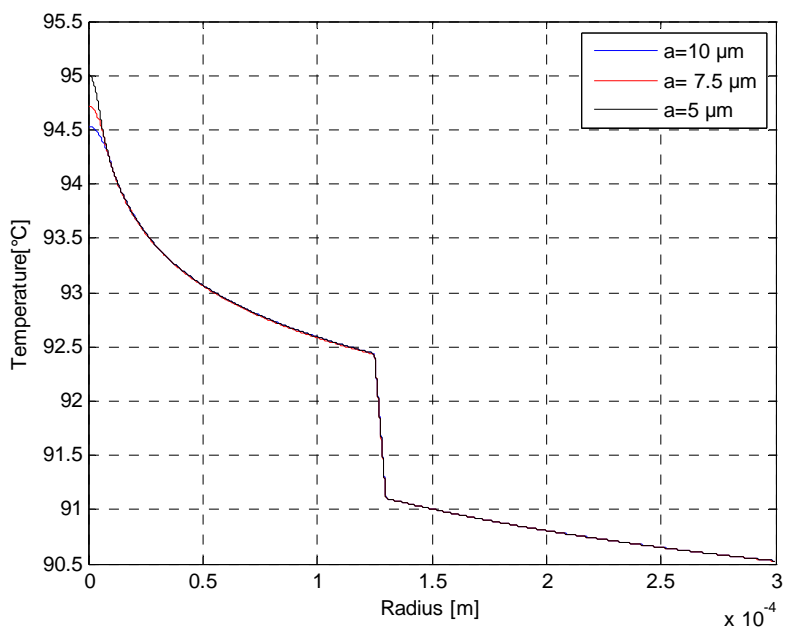

(a)

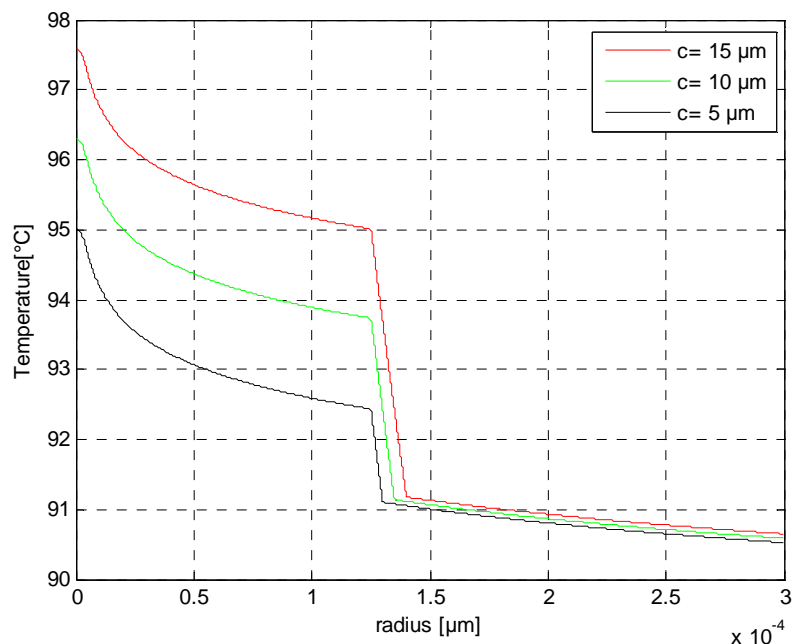

(b)

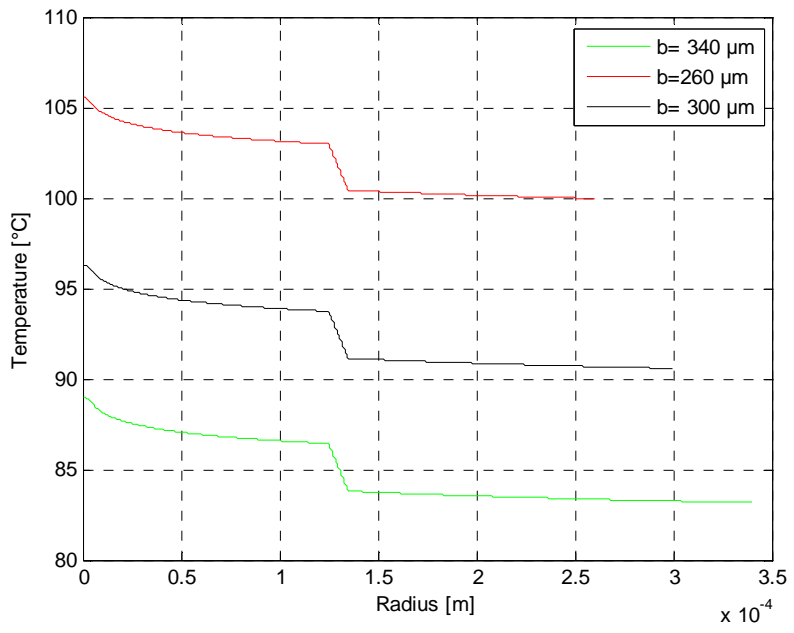

(c)

Figure 6. Temperature distributions in Bi-directional pump mode at (a) The fiber input side for different core radius; (b) The fiber input side for different air-clad width; and (c) The fiber input side for different cladding radius in bi-directional pump mode. 


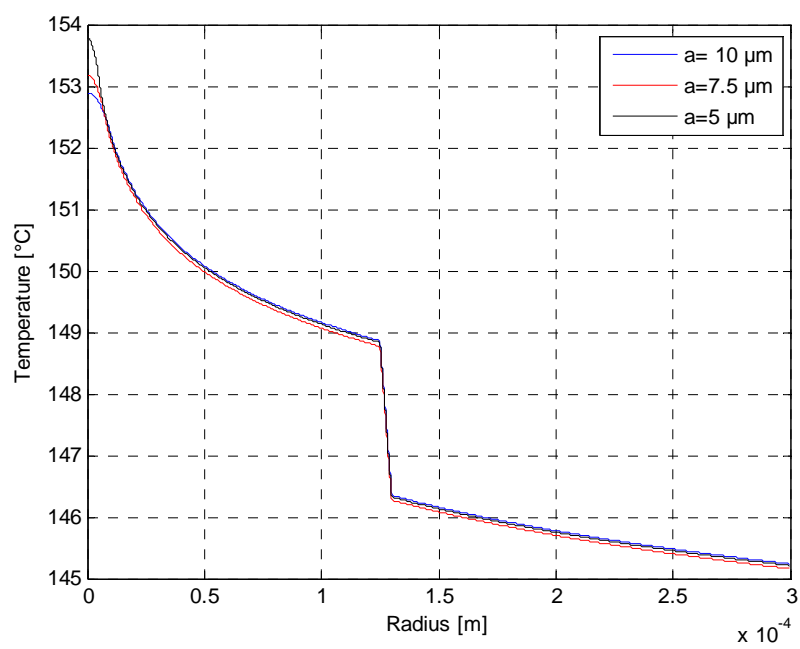

(a)

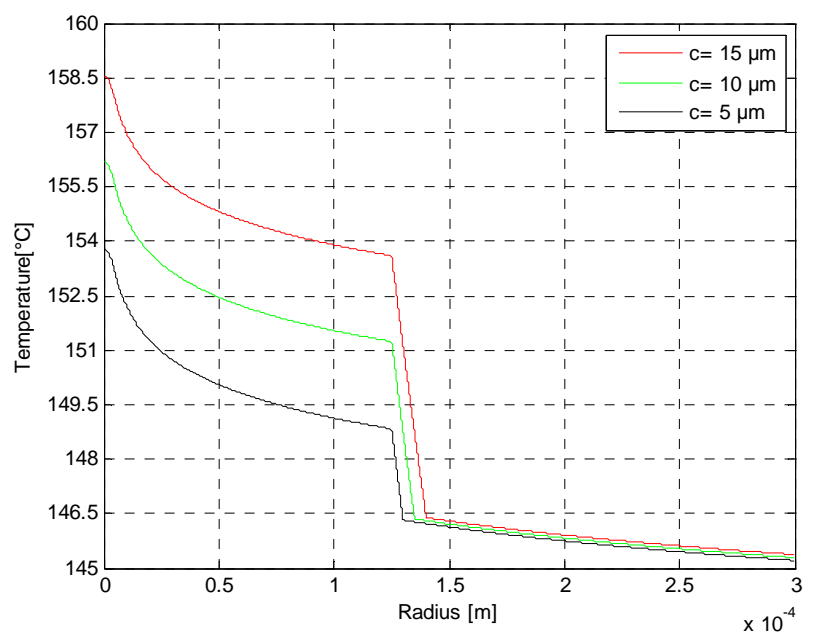

(b)

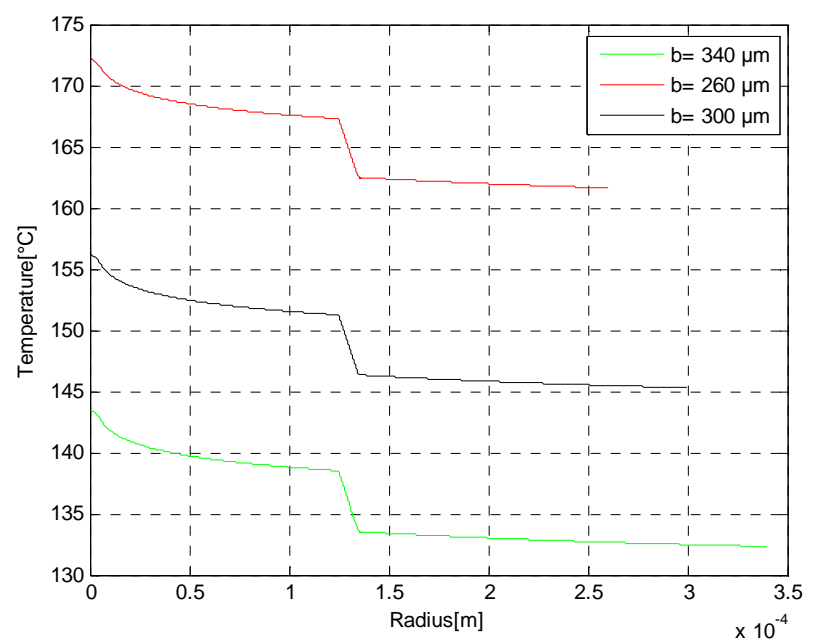

(c)

Figure 7. Temperature distributions in the Backward pump with $R_{p 1}=0$. At (a) The fiber axis output side for different core radius; (b) The fiber input side for different air-cladding width; and (c) The fiber output side for different cladding radius.

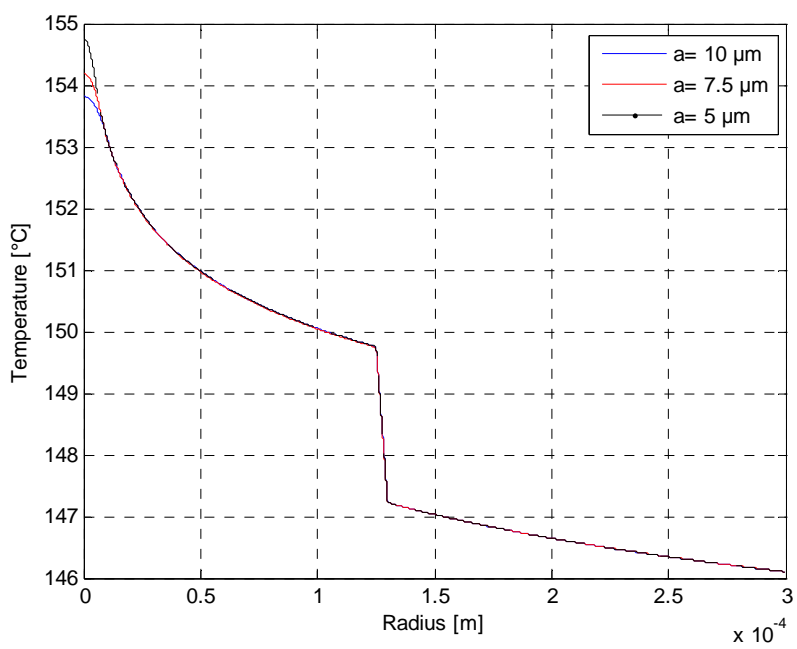

(a)

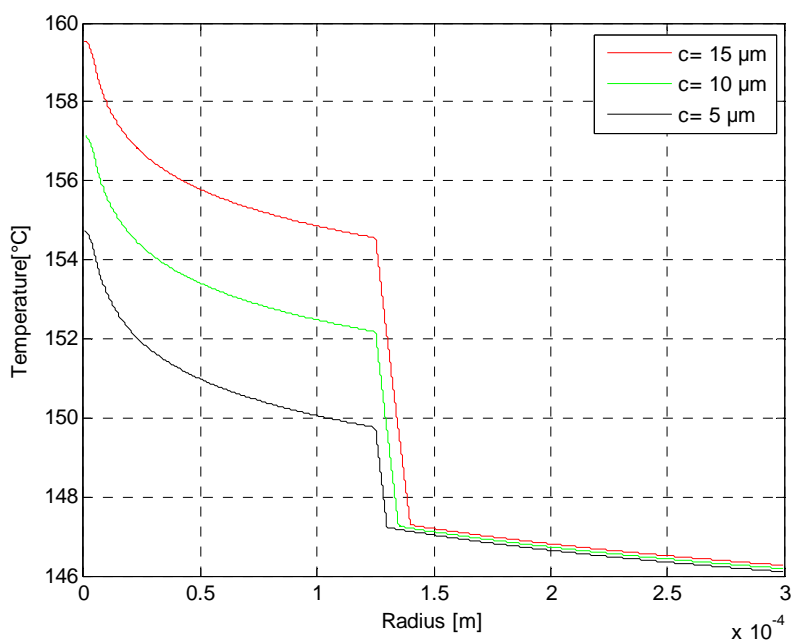

(b)

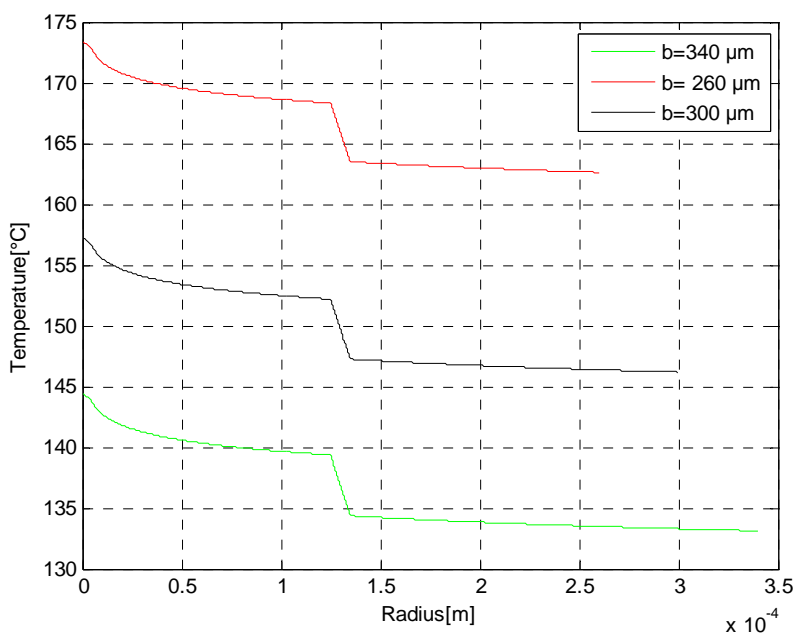

(c)

Figure 8. Temperature distributions in the Backward pump with $R_{p 1}=0.98$ at (a) The fiber axis output side for different core radius; (b) The fiber input side for different air-clad width; and (c) The fiber output side for different cladding radius. 


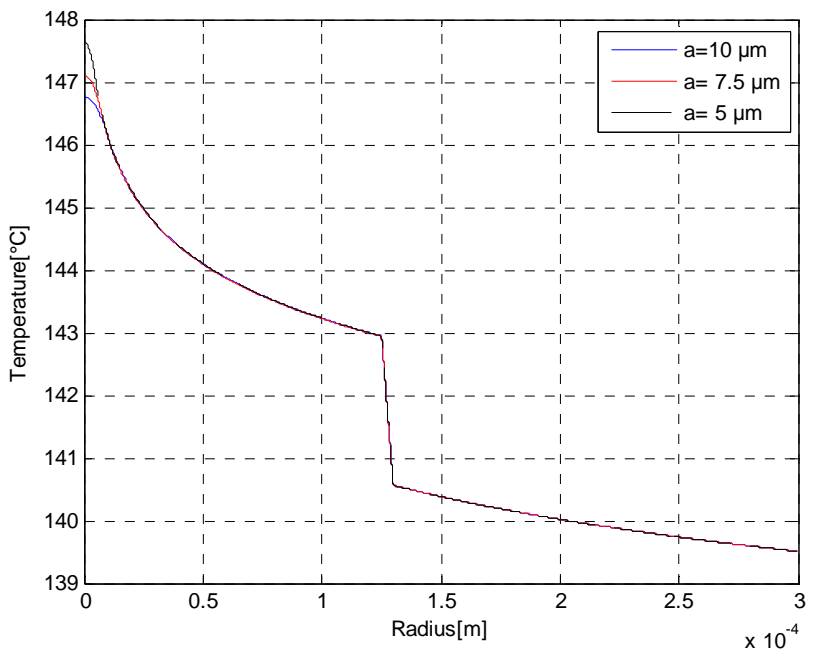

(a)

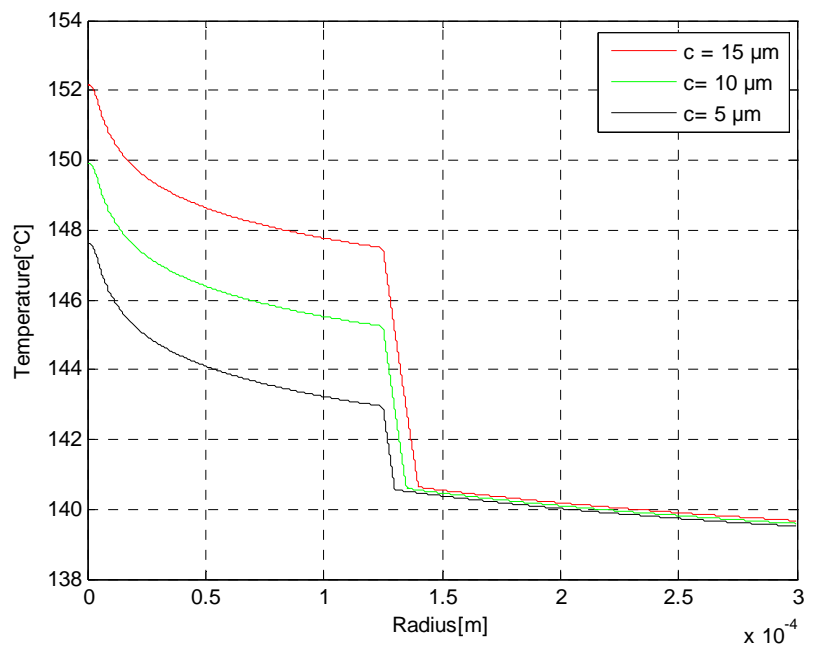

(b)

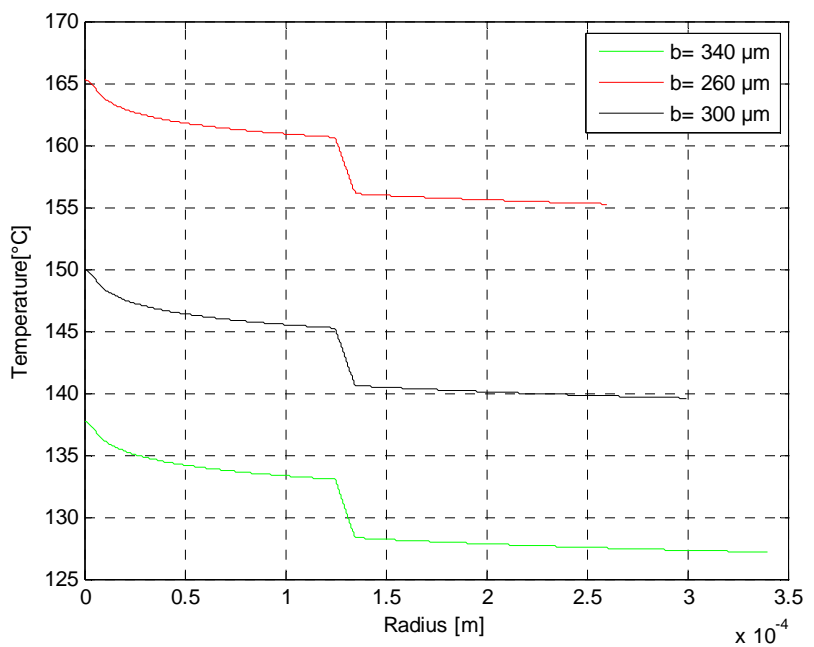

(c)

Figure 9. Temperature distributions in the forward pump with $R_{p 1}=0$ at (a) The fiber axis input side for different core radius; (b) The fiber input side for different air-clad width; and (c) The fiber input side for different cladding radius.

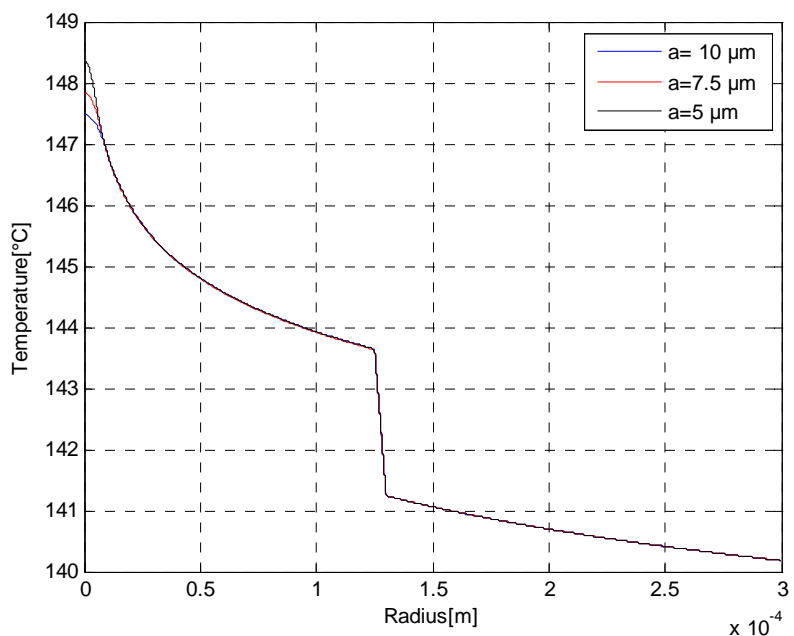

(a)

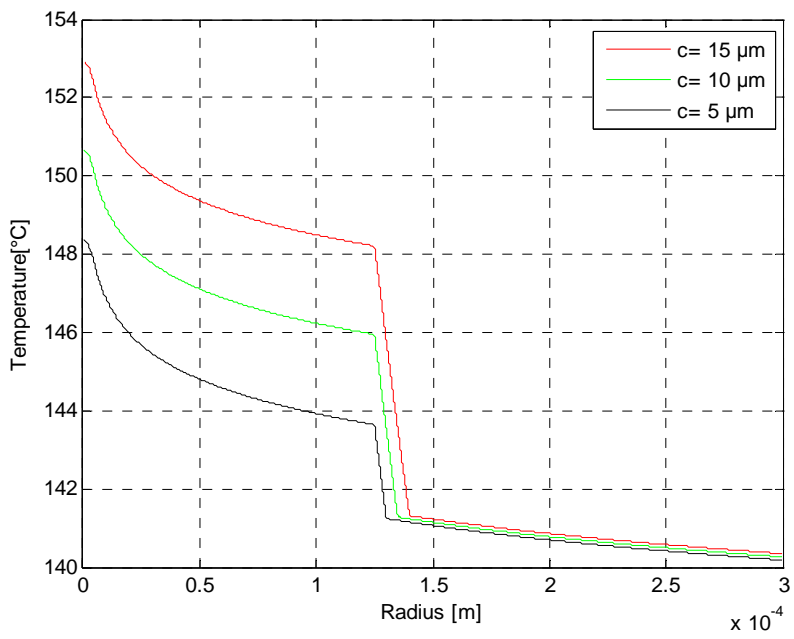

(b)

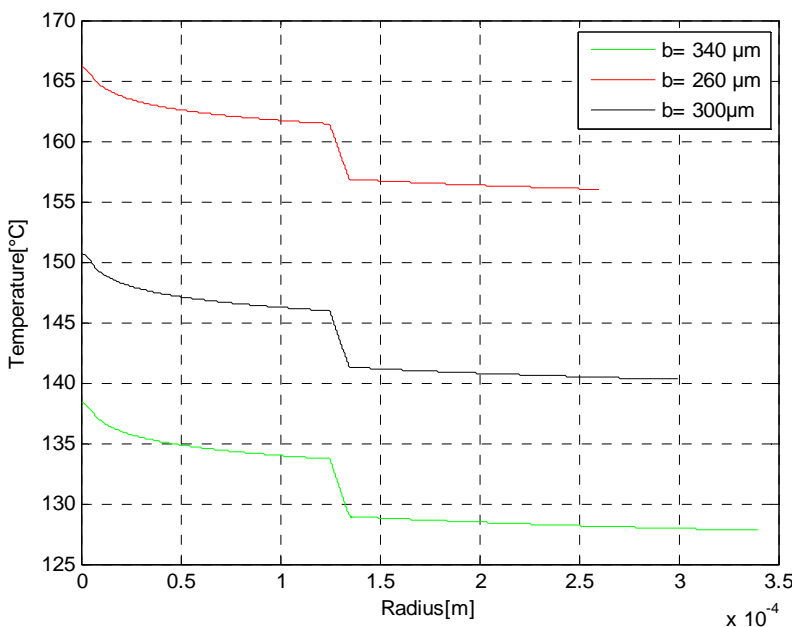

(c)

Figure 10. Temperature distributions in the Forward pump with $R_{p 1}=0.98$ at (a) The fiber axis input side for different core radius; (b) The fiber input side for different air-clad width; and (c) The fiber input side for different cladding radius. 
pation and the temperature distribution for different pumps schemes. The results show that the temperature distribution for bidirectional pump mode is more even than that the forward pump with reflection $R_{p 2}$ and backward pump with reflection $R_{p 1}$. These two latter schemes are in agreement for saving pump powers and YDCF length. Moreover, the forward pump with $R_{p 1}=0.98$ and the backward pump with $R_{p 2}=0.98$ in less length resulting in high output power are thermally acceptable. Further experimental and theoretical studies are needed to explain the temperature distribution of fiber laser with different pump schemes.

\section{REFERENCES}

[1] J. Limpert, A. Liem, H. Zellmer and A. Tunnerman, "500 W Continuous-Wave Fiber Laser with Excellent Beam Quality," Electronics Letters, Vol. 39, No. 8, 2003, pp. 645-647. http://dx.doi.org/10.1049/el:20030447

[2] Y. Wang and C.-Q. Xu, "Modeling and Optimization of Q-Switched Double-Clad Fiber Laser," Applied Optics, Vol. 45, No. 9, 2006, pp. 2058-2071.

[3] R. Paschotta, J. Nilsson, A. C. Tropper and D. C. Hanna, "Ytterbium-Doped Fiber Amplifiers," IEEE Journal of Quantum Electronics, Vol. 33, No. 7, 1997, pp. 10491056. http://dx.doi.org/10.1109/3.594865

[4] Y. Jeong, J. K. Sahu, D. N. Payne, et al., "YtterbiumDoped Large-Core Fiber with $1.36 \mathrm{KW}$ of ContinuousWave Output Power," Optics Express, Vol. 12, No. 25, 2004, pp. 6088-6092. http://dx.doi.org/10.1364/OPEX.12.006088

[5] D. Gapont, "Quasi-Single-Mode Fiber Laser Nears $2 \mathrm{~kW}$ Output with High-Quality Beam," Laser Focus World, Vol. 41, No. 6, 2005, pp. 9-11.
[6] S. Baek, D. B. S. Soh, Y. Jeong, J. K. Sahu, J. Nilsson and B. Lee, "Acladding-Pumped Fiber Laser with PumpReflecting Bragg Grating," IEEE Photonics Technology Letters, Vol. 16, No. 2, 2004, pp. 407-409. http://dx.doi.org/10.1109/LPT.2003.823135

[7] I. Kelson and A. Hardy, "Srongly Pumped Fiber Lasers," IEEE Journal of Quantum Electronics, Vol. 34, No. 9, 1998, pp. 1570-1577. http://dx.doi.org/10.1109/3.709573

[8] L. Shang, "Comparative Study of the Output Characteristics of Ytterbium-Doped Double-Clad Fiber Lasers with Different Pump Schemes," Optik, Vol. 122, No. 21, 2011, pp. 1899-1902.

[9] J. F. Li, "Theoretical Analysis of the Heat Dissipation Mechanism in High Power Photonic Crystal Fiber Laser," Optik, Vol. 121, No. 13, 2010, pp. 1243-1250. http://dx.doi.org/10.1016/j.ijleo.2009.01.015

[10] W. M. Rohsenow, J. P. Hartnett and Y. I. Cho, "Handbook of Hear Transfer," McGraw-Hill, New York, 1998.

[11] B. David, "Therma, Stress, and Thermo-Optic Effects in High Average Power Double-Clad Silica Fiber Lasers," IEEE Journal of Quantum Electronics, Vol. 37, No. 2, 2001, pp. 207-217.

[12] J. F. Li, "Theoretical Analysis and Heat Dissipation of Mid-Infrared Chalcogenide Fiber Raman Laser," Optics Communications, Vol. 284, No. 5, 2011, pp. 1278-1283. http://dx.doi.org/10.1016/j.optcom.2010.10.062

[13] P.-X. Li and C. Zhu, "Theorecal and Experimental Investigation of Thermal Effects in a High Power $\mathrm{Yb}^{3+}$-Doped Double-Clad Fiber Laser," Optics and Laser Technology, Vol. 40, No. 2, 2008, pp. 360-364. http://dx.doi.org/10.1016/j.optlastec.2007.06.011

[14] J. P. Holman, "Heat Transfer," 8th Edition, McGraw-Hill, New York, 1997. 